\title{
On predictions and laws in biological evolution
}

\author{
Is biology able to erect general laws and develop inductive predictions, as \\ usual in physics or chemistry?
}

\author{
Valentí Rull \\ Botanic institute of Barcelona (IBB-CSIC), Barcelona, Spain
}

As is well known, science uses evidence-based inductive reasoning to build theories, principles and laws. A common type of inductive reasoning is generalization, i.e., the projection into a broader context of the conclusions drawn from one or a few case studies. The reliability of generalizations depends upon the representativeness and the formal validation of the selected case studies, which is usually performed by hypothesis testing. Another usual type of inductive reasoning is prediction, which uses past and present observations to develop principles and laws that allow anticipation of future outcomes. The reliability of predictions is given by the actual accomplishment of the anticipated situation. It is interesting to note that generalizations are based on proposals that emerge from the analysis of empirical evidence, whereas predictions are formulated before the desired empirical evidence, which is actually the target of the prediction, is obtained.

The American philosopher of science Peter Lipton (2005) pointed out that we are commonly more impressed by predictions than by "accommodations", as he called hypothesis testing procedures. To illustrate this situation, Lipton used the process of discovery of Halley's Comet. In 1705, the British astronomer Edmond Halley proposed that the comets observed in 1531, 1607 and 1682 were actually a single comet with a periodic elliptical orbit. This view did not have much impact within the scientific community. However, when Halley's prediction of the return of the comet in 1758 was actually confirmed, the European intellectual world widely accepted the existence of a single comet, which was named Halley's Comet. Halley's prediction may seem straightforward, even trivial, considering the characteristic periodicity of 75 years in previous observations. However, it was the predictive success, rather than prior observations, that convinced the scientific community.

Physics is among the strongest branches of science - together with chemistry and mathematics - with regard to the generality and accuracy of predictions. Biology seems to still be in its infancy in this sense, and the search for regularities leading to potential generalizations is the most common approach (Dodds, 2009). This is due in part to the high level of complexity of the living world itself, its evolutionary change over time and its ecological relationships with the surrounding environment. As emphasized by the German evolutionary biologist Ernst Mayr (2004), these intrinsic and unique features of living beings, which are intimately associated with the existence of the genetic code, clearly differentiate biology from other natural sciences and make the fundamental laws of physics and chemistry insufficient to understand the living world. Many scientists do not agree with this statement and defend a reductionist approach, according to which any phenomenon occurring in the Universe may ultimately be reduced to the action of the fundamental laws of matter. This is an old debate that is beyond the scope of this essay and has already been discussed in a former paper (Rull, 2012).

Another factor that might have contributed to the dominance of generalization, rather than predictive, approaches in biology could be linked to the attitude of biologists themselves, a topic that is discussed by Dodds (2009). Modern global databases and modeling techniques have contributed to increasing the interest in biological predictions, mainly in the field of 
potential ecological responses to ongoing climate change. However, other biological disciplines still have not developed similar tools, as is the case for evolution, mainly due to the contingent and unpredictable nature of the evolutionary process. In the present state of knowledge, predictions about the future of evolution are unwarranted, even in the case of single lineages. Efforts in this sense have largely been concentrated on our own species, with an emphasis on human manipulations such as genetic engineering or invasive technological applications (Chan, 2008).

However, it should be noted that inductive prediction may be a more general concept concerned not only with the future, in chronological terms, but also with the need for anticipating the findings suitable for filling current knowledge gaps. In other words, we could be interested in predicting the existence of entities, the evidence for which is still lacking, that would be needed to understand observed phenomena that remain unexplained. In the absence of empirical evidence for these entities, prediction is the most feasible solution. Prediction is a common feature in physics, as shown in cases such as the Higgs boson or interstellar dark matter, just to name a few.

\section{Physical and chemical predictions and laws}

The existence of the Higgs boson was predicted in the 1960s as a necessity for the Standard Model of particle physics (SMPP) to be valid, even in the presence of some subatomic particles having mass. Of the four fundamental forces of nature - i.e., gravity, electromagnetic force, weak atomic force and strong nuclear force - electromagnetic and weak forces have been unified into electroweak force, which is responsible for electricity, magnetism, light and some types of radioactivity. The equations of the SMPP accurately describe the electroweak force assuming that the particles involved do not have mass, which is true for photons but not for particles such as $\mathrm{W}$ and $\mathrm{Z}$ bosons. This inconsistency was solved by proposing the existence of the Higgs field, named after the British physicist Peter Higgs, which would grant mass to any particle interacting with it. The particle associated with this field was tentatively called the Higgs boson, and its mass, charge and spin were estimated with the SMPP. In 2012, after five decades of experiments, this particle was discovered in the CERN Large Hadron Collider (LHC), and Peter Higgs, together with his Belgian colleague François Englert, was awarded the Nobel Prize in 2013 for this fundamental finding (https://home.cern/science/physics/higgs-boson).

Other physical predictions await empirical confirmation, as is the case for the existence of interstellar dark matter. Astrophysical observations of gravitational fields of many galaxies are difficult to explain by current gravitational theories unless more matter is present than can be seen. This has led to the prediction that an unknown form of matter is abundant in the universe, which has been called "dark" because it does not interact with the electromagnetic field; that is, it does not absorb, reflect or emit electromagnetic radiation and is therefore difficult to detect. However, dark matter particles are assumed to carry energy and momentum, and hence, they might theoretically be detectable in the LHC (https://home.cern/science/physics/dark-matter).

In elemental chemistry, the periodic table is another amazing example of successful predictive inference. The background law behind this table is the periodic law, formulated in 1869 by the Russian chemist Dimitri Mendeleev, according to which the properties and atomic structures of the chemical elements are a periodic function of their atomic numbers (the number of protons in the nucleus), which uniquely identify each element. Mendeleev organized the chemical elements known at the time in a table according to their atomic numbers and observed gaps, which he considered to correspond to still unknown elements, and used the positions of these gaps to predict the atomic composition of the missing elements according to 
the periodic law. With time, these predicted elements have progressively been identified to conform to the current periodic table, which is a fundamental scientific achievement.

A common feature of these predictions is the axiomatic nature of the fundamental background laws, in this case the SMPP, gravitational laws and the periodic law of elements. Predictions of new subatomic particles, new chemical elements or still undetected interstellar matter are necessary to sustain these fundamental laws, and each time that a prediction is accomplished, these laws are reinforced. In other words, physicochemical laws are viewed as immutable rules, and knowledge advances by finding the evidence needed to fulfill these laws. A corollary is that the predictive approach seems better developed in scientific disciplines with wellestablished fundamental laws.

\section{Biological laws}

In biology, there is a lack of consensus on the existence of general laws. A number of biologists and philosophers, including Ernst Mayr (2004), contend that there are no laws in biology, due to the contingent and unpredictable nature of living beings and their evolution. Others rely on finding regularities in biological contingency potentially leading to general laws or tentatively formulate biological laws, usually with a strong physical component, with a recurrent emphasis on thermodynamic laws (Trevors \& Saier, 2010; Brandon, 2013). Claiming that biology fits with the laws of thermodynamics cannot be considered a biological law, by no means. It is actually a physical law. Conversely, the tendency of evolution to minimize entropy (Mayr, 2004; Rull, 2011), which represents a challenge for the universality of the second law of thermodynamics, may be the embryo for a potential biological law. However, the truth is that generally accepted laws specific to the biological world are still lacking. However, most theorists agree in a paramount and unique biological singularity: all living organisms and the complex spatial, temporal, functional and ecological diversity patterns they generate emerge by evolution.

It follows that if general laws specific to biology actually exist, they would likely have to be derived from the theory of evolution, which constitutes a fundamental and distinctive biological foundation. Therefore, in the absence of biological laws, the ability of the modern theory of evolution, along with recent developments in genomics and molecular phylogenetics, to support inductive predictions is worth exploring. As noted above, predicting the future of evolution is still unworkable; however, the possibility of predictions similar to the Higgs boson, dark matter or the "hidden" elements of the periodic table might eventually be possible. This endeavor should not be confused with a reductionist approach aiming to apply physical laws to biology; it is merely a conceptual and methodological comparison.

\section{Possible evolutionary predictions}

Whether biological evolution progresses gradually or by leaps is an old debate. An example is the controversy between phyletic gradualism (PG) and punctuated equilibrium (PE). The PG concept proposes that evolution is dominated by slow and gradual processes of transformation of one species into another (anagenesis) under the action of natural selection on species' populations. This view has been considered by some a consequence of Darwin's concept of natural selection. In contrast, PE contends that most evolution occurs in the form of rapid speciation events that split one species into two distinct species (cladogenesis), followed by long phases without significant evolutionary changes (stasis). The PE concept was proposed by the American paleontologists Stephen Jay Gould and Niles Eldredge (1977), who argued that gradual change is not observed in the fossil record, which is dominated by long-ranging "static" fossil morphologies. The defenders of PG attributed these observations to the incompleteness and fragmentary nature of the fossil record. 
The fossil record has traditionally been the main source of empirical evidence for evolution and - like the first periodic table of Mendeleev, which was still incomplete - maybe it could be used to predict the fossil evidence needed to adhere to either the PG or PE view. Under the PG rule, the fossil record should contain the whole range of intermediate morphologies representing the gradual anagenetic process. Furthermore, PG should eventually be able to predict these forms, as Mendeleev did with the "hidden" elements. There are three main limitations preventing such prediction: 1) general or particular evolutionary laws of morphological change, similar to the periodic law of the elements, are lacking; 2 ) fossil morphology is only part of the actual living species, usually the harder parts, and eventual morphological changes in other already lost parts are unnoticed; and 3) fossil morphology is one of the possible phenotypic expressions of the species' genotype, which is the actual evolutionary material. Even in the case that PG would be able to predict the fossils to be found under this evolutionary mode, finding the necessary empirical evidence would be prevented by the intrinsic incompleteness of the morphological fossil record.

In contrast to PG, which is grounded in natural selection acting on species' populations, $P E$ is essentially based on the actual structure and composition of the available morphological fossil record. Therefore, by definition, PE is comfortable with the fossil record as is and does not seem to have any predictions to do in this respect. At present, the PG and PE proposals are considered two extreme views within the general context of evolutionary rates, which are not constant but variable across species (Futyma, 2000). It is worth noting that our eventual ability to accurately predict the fossils needed to fit with any evolutionary model, regardless of the involved rates, might provide the bases for the formulation of evolutionary laws and, therefore, for predicting future evolution. However, in the current state of knowledge, the limitations mentioned above seem insurmountable. Therefore, the situation is conceptually more similar to the astrophysical proposal of dark matter, as in addition to fossil morphotypes, a huge amount of still hidden "dark evolutionary matter" is needed to properly understand biological evolution. The main difference is that physicists know what the target evidence is (the predicted dark matter particles) and the suitable methodology for finding it (particle acceleration), whereas biologists seem to ignore what are we looking for.

Fortunately, research is in constant progress, and our knowledge of the fossil record has greatly improved since the 1970s and 1980s, when the PG-PE debate was glowing. Recently, sophisticated analytical methods have been developed to identify the genome, or parts of it, preserved in fossil remains of many species and in sedimentary archives. This has added a highly significant amount of dark evolutionary matter to the morphological fossil record. Importantly, this additional matter includes the DNA of fossil organisms (also called ancient DNA or aDNA), which is the evolutionary subject par excellence and, hence, the fundamental evolutionary matter. This discovery inaugurated so-called paleogenomics, which would make possible the reconstruction of the evolutionary trends themselves, that is, the genetic changes experienced by the species over time. Other types of molecular biomarkers useful in evolution, notably proteins and lipids, have also been found in the fossil record. The main challenge is preservation, due to the labile nature of the involved biomolecules. Despite this, advances in the study of ancient biomolecules made during recent decades have transformed our understanding of the evolutionary history of life on Earth. Paleogenomics has already provided direct insights into evolution that few would have predicted less than a decade ago (Cappellini et al., 2018). Therefore, this particular field of knowledge progresses by accumulation of empirical evidence, rather than by its prediction, as in the cases of the missing elements of the periodic table and interstellar black matter. 
The genome of extant organisms could be considered a compendium of their evolutionary history, similar to a miniature fossil record. The recent development of DNA molecular methods of analysis has allowed the detailed reconstruction of the phylogenetic history of living beings using the genome, which is evolutionary material itself. In this way, we might be able to know the genetic features of still unknown ancestors of extant organisms and, possibly, to predict how they would have looked. This procedure is somewhat similar to the prediction of the existence of the Higgs boson in that we would be able to anticipate the genotypic and, eventually, the phenotypic features of the still undiscovered predecessors or "evolutionary bosons". In this way, we would be more conscious of what are we looking for in the fossil record, which could be useful for planning research, just as physicists adjust the parameters of the particle accelerators when they are aware of the physical properties of their predicted targets. Some of the abovementioned drawbacks still apply in this case, notably the fragmentary nature of the fossil record and the problems associated with the phenotypic expression of genotype features. However, this possibility seems to be somewhat similar to the physicochemical predictions discussed above. The main difference is that we would need a different evolutionary boson for each lineage and each time period considered, which would make the prediction largely empirical rather than theoretical. Indeed, the existence of eventual evolutionary bosons is not based on a fundamental evolutionary law but on phylogenetic reconstructions, which are probabilistic hypotheses that vary over time with progressive methodological improvements (Hawkins, 2006).

\section{Hypothesis and predictions}

Hypotheses may be formulated as predictions, but in the absence of underlying laws, they are still hypotheses rather than inductive predictions. For example, predicting that $\mathrm{PE}$, rather than $P G$, explains speciation in a given taxon under study is not really a prediction, in the physical sense, similar to predicting the existence of the Higgs boson or dark matter. In the case of PE vs. PG, the research target is not empirical evidence that emerges from a fundamental law (predictive induction) but an explanation for a previously observed phenomenon (hypothesis testing). The word "predict" does not transform the process of hypothesis testing into a case of inductive prediction, as the underlying concepts are different. So-called predictive modeling may also be considered a process of hypothesis testing and further generalization. In this case, models are the hypotheses to be tested. If model outcomes recurrently fit with actual observations, then these models are considered robust enough to explain or reproduce the empirical evidence and may be generalized into predictive tools. Eventual deviations from the predicted outputs lead to modifications to adjust models according to new observations in a process of continuous model improvement, which is analogous to the continuous hypothesis revision typical of accommodating procedures (sensu Lipton, 2005). The same is true, for example, for the abovementioned case of DNA phylogenetic reconstructions. The difference with inductive prediction, as used in physics, is that physical laws are considered immutable, and eventual prediction failures are attributed to methodological or technological deficiencies that hinder finding the evidence predicted by these laws. This leads to continuous technological improvements rather than the constant reconsideration of laws.

\section{Which is better?}

There is nothing intrinsically good or bad in inductive prediction and accommodating generalization per se, and different scientific disciplines may have diverse procedures, depending on the nature of the part of the world they are trying to understand. The differences between living beings and dead matter are not in the world of subatomic particles or elemental composition, as these domains and their dynamics are common to both, but in the emergent properties generated at higher organization levels. Biological evolution, which 
uniquely characterizes living organisms, is possible because of the existence of the molecular genetic code and its random mutation ability. Then, the lower organization level at which it is possible to differentiate living from dead matter is the molecular level. Above, there is the organismic level, with emergent properties also unique to living beings, such as birth, reproduction and death. Still above, there is the ecological level, characterized by the relationships between species' communities and between those communities and the external environment, which guide selection and are also unique to living systems. Therefore, if fundamental biological laws actually exist, they should be sought at these organization levels rather than in the world of particle physics or elementary chemistry. The difficulty of finding biological laws may be a consequence of properties such as the contingence and the unpredictability generated by the structural and functional hypercomplexity of these higher organization levels.

\section{The incommodity of life}

When considering our planet as an element of astronomical systems of higher hierarchy, the phenomenon of life seems to be lost into a world of physical forces again. Regardless of the possibility of extraterrestrial life, the dynamics of the universe are ruled by large-scale physical laws such as gravity. Therefore, life may be considered an intermediate phenomenon between the molecular and astronomical levels, for which fundamental laws are elusive, despite the efforts of a number of biologists to find them and a legion of physicists to develop a "theory of everything" (Hawking \& Mlodinov, 2010) that reduces the phenomenon of life to fundamental physical laws.

It could be asked why biologists need to imitate conceptual methodologies of other scientific disciplines such as physics, chemistry or mathematics and compulsively seek to find fundamental biological laws. Why do we need to change the paths of biological research and transform it into a science based on inductive predictions, rather than on hypothesis testing and generalization?. Advances made in biological knowledge using these accommodating procedures are evident. What if, after all, fundamental laws only work for submolecular and astronomical worlds and life is a disturbing anomaly in between?

\section{References}

Brandon, R.N. (2013) Does biology have laws? The experimental evidence. Philosophy of Science 64, S444-S457.

Cappellini, E., Prohaska, A., Racimo, F., Welker, F., Pedersen, M.W., Allentoft, M.E., et al. (2018) Ancient biomolecules and evolutionary inference. Annual Review of Biochemistry 87, 1029-1060.

Chan, S. (2008) Humanity 2.0? EMBO Reports 9, S70-S74.

Dodds, W.K. (2009) Laws, Theories, and Patterns in Ecology. University of California Press, Berkeley.

Futuyma, D. (2005) Evolution. Sinauer, Sunderland.

Gould, S.J., Eldredge, N. (1977) Punctuated equilibria: the tempo and mode of evolution reconsidered. Paleobiology 3, 115-151.

Hawking, S., Mlodinov, L. (2010) The (elusive) theory of everything. Scientific American 303, 68-71.

Hawkins, J.A. (2006) Using phylogeny to investigate the origins of the Cape flora: the importance of taxonomic, gene and genome sampling strategies. Diversity and Distributions 12, 27-33.

Lipton, P. (2005) Testing hypotheses: prediction and prejudice. Science 307, 219-221.

Mayr, E. (2004) What Makes Biology Unique? Cambridge University Press, Cambridge. 
Rull, V. (2012) Time, evolution and physical reductionism. EMBO Reports 13, 181-185.

Trevors, J.T., Saier, M.H. (2010) Three laws of biology. Water, Air and Soil Pollution 205, S-87S89. 